\title{
Offline and online scheduling of electric vehicle charging with a minimum charging threshold
}

\author{
Martijn H. H. Schoot Uiterkamp, Thijs van der Klauw, Marco E. T. Gerards and Johann L. Hurink \\ Faculty of Electrical Engineering, Mathematics and Computer Science \\ University of Twente, Enschede, the Netherlands \\ Email: \{m.h.h.schootuiterkamp, t.vanderklauw, m.e.t.gerards, j.1.hurink\}@utwente.nl
}

\begin{abstract}
The increasing penetration of electric vehicles (EVs) requires the development of smart charging strategies that accommodate the increasing load of these EVs on the distribution grid. Many existing charging strategies assume that an EV is allowed to charge at any rate up to a given maximum rate. However, in practice, charging at low rates is inefficient and often even impossible. Therefore, this paper presents an efficient algorithm for scheduling an EV within a decentralized energy management system that allows only charging above a given threshold. We show that the resulting optimal EV schedule is characterized by an activation level and a fill-level. Moreover, based on this result, we derive an online approach that does not require predictions of uncontrollable loads as input, but merely a prediction of these two characterizing values. Simulation results show that the online algorithm is robust against prediction errors in these values and can produce near-optimal online solutions.
\end{abstract}

\section{INTRODUCTION}

The number of EVs is rapidly increasing. Smart control of the charging of these EVs is required in order to reduce energy losses, preserve grid assets and prevent blackouts and overloading [1]. One common control paradigm for EV charging is decentralized energy management (DEM). In DEM, devices are individually scheduled and subsequently coordinated by means of a central controller. Often, the devices are scheduled based on a steering signal issued by the central controller such as energy prices (also known as demand response [2]) and target profiles that explicitly specify the amount of energy that should be consumed (see, e.g., [3]).

Many algorithms for scheduling EV charging in DEM exist (for an overview, see [4]). Most of these algorithms assume that the EV can charge at any rate between zero and a given maximum rate. However, in practice, this is not always possible due to restrictions on the minimal charging current implemented by EV battery manufacturers (see, e.g., [5], [6]). Furthermore, charging an EV at a low rate reduces the efficiency of the charging process (see, e.g., [7]). Therefore, it makes sense to allow only charging above a given minimum rate to prevent inefficient charging. Only a few works consider such a minimum-threshold constraint (see, e.g., [8]).

In DEM, the device objective is often based on the power profile of a household, the base load. However, in practice, the base load is not known beforehand. To solve this problem, most EV charging algorithms use predictions of the base load as input for the offline scheduling algorithm. However, it is very hard to obtain accurate base load predictions (see, e.g., [9]). Therefore, other approaches are needed to solve this issue.
This paper presents an efficient algorithm for scheduling an $\mathrm{EV}$, suitable for DEM, that takes into account the minimumthreshold constraint as stated above and that flattens the combined base load and EV profile as much as possible. We show that the optimal EV schedule can be characterized by two values that function as an activation level and fill-level respectively. Based on this characterization, we also derive an online algorithm for the case where the base load profile is unknown. This algorithm uses a prediction of only the two characterizing values, rather than a prediction of the entire base load profile, to schedule an EV. Simulation results show that this algorithm is robust against prediction errors in the two characterizing parameters and yields near-optimal solutions.

The remainder of this paper is structured as follows. In Section II, we formally introduce the EV charging problem that we study in this paper and analyze the hardness of this problem. Section III provides an analysis of the structure of optimal solutions to the scheduling problem and Section IV presents a polynomial time algorithm to solve the problem. In Section V, we derive an algorithm to solve the online version of the problem based on the algorithm of Section IV. In Section VI, we conduct a simulation study to validate our approach and Section VII provides our conclusions.

\section{Problem Statement}

In this section, the EV charging problem that we study in this paper is introduced. Section II-A provides the formal problem statement and Section II-B discusses the complexity of this problem and several of its special cases.

\section{A. Problem statement}

We assume that the charging window of the EV (the time period wherein it is available for charging) is known. We discretize the problem, meaning that we divide the charging window into $T$ time intervals $\mathcal{T}=\{1, \ldots, T\}$. We denote by $\vec{x}=\left(x_{t}\right)_{t \in \mathcal{T}}$ the charging profile, where the variable $x_{t}$ denotes the energy volume that is charged during interval $t$. Moreover, we assume that the amount $C$ that needs to be charged within the charging window is known. For each time interval $t \in \mathcal{T}$, let $X_{t}^{\min }>0$ and $X_{t}^{\max }$ be the given minimum and maximum allowed charging rates for that interval $t$. To model the decision whether or not to charge during an interval $t$, we introduce a binary variable $y_{t}$ that is 1 if the EV charges during $t$ and 0 otherwise. The objective is to charge the EV such that the combined base load and charging profile is flattened 
as much as possible. We denote this base load profile by $\vec{p}=\left(p_{t}\right)_{t \in \mathcal{T}}$. The objective of load profile flattening can be modeled by minimizing the 2 -norm of $\vec{p}+\vec{x}$. For convenience and without loss of generality, we choose to minimize the square of this 2-norm.

This leads to the following optimization problem:

$$
\begin{aligned}
& (P): \min _{\vec{x}} \sum_{t=1}^{T} f_{t}\left(x_{t}\right)=\sum_{t=1}^{T}\left(p_{t}+x_{t}\right)^{2} \\
& \text { s.t. } \sum_{t=1}^{T} x_{t}=C \\
& X_{t}^{\min } y_{t} \leq x_{t} \leq X_{t}^{\max } y_{t} \\
& y_{t} \in\{0,1\} \\
& \forall t \in \mathcal{T}, \\
& \forall t \in \mathcal{T} \text {. }
\end{aligned}
$$

Throughout this paper, we assume that Problem $(P)$ is feasible and, without loss of generality, that the values $p_{t}$ are distinct and that the intervals are sorted according to descending values of $p_{t}$, i.e., we assume that $p_{1}>p_{2}>\ldots p_{T}$.

Note that the above formulation allows us to model the objective of following a given target profile $\vec{q}$ as closely as possible by setting $\vec{p}=-\vec{q}$. Furthermore, we can include a linear term $c_{t} x_{t}$ into the objective without changing the problem structure by choosing $f_{t}\left(x_{t}\right)=\left(p_{t}+x_{t}+\frac{1}{2} c_{t}\right)^{2}$, thus taking $p_{t}+\frac{1}{2} c_{t}$ as the new "base load" for interval $t$. This is because $\left(p_{t}+x_{t}+\frac{1}{2} c_{t}\right)^{2}=\left(p_{t}+x_{t}\right)^{2}+c_{t} x_{t}+c_{t} p_{t}+\frac{1}{4} c_{t}^{2}$ and adding a constant to the objective function does not change the optimal solution and set of feasible solutions.

Problems of type $(P)$ exist in the literature as knapsack problems with setups [10] or semi-continuous knapsack problems [11]. However, to the best of our knowledge, no literature exists on such problems with quadratic objective functions, except for a preliminary analysis of Problem $(P)$ in [12].

\section{B. Complexity analysis}

In this section, we analyze the complexity of Problem $(P)$. Note that this problem without any restrictions on the bounds $X_{t}^{\min }$ and $X_{t}^{\max }$ is NP-hard, which can be shown using a reduction from the subset-sum problem. In fact, it turns out that the problem is NP-hard even for a fixed $\vec{X}^{\max } \in \mathbb{R}_{+}^{T}$.

Lemma 1. Problem $(P)$ is NP-hard for any $\vec{X}^{\max } \in \mathbb{R}_{+}^{T}$.

Proof: We prove that $(P)$ is NP-hard by deriving a polynomial-time reduction from the subset-sum problem to $(P)$. For this, fix $\vec{X}^{\max }$ and let $\mathcal{I}_{\text {SSP }}$ be an instance of the subset-sum problem wherein $\mathcal{S}:=\left\{S_{1}, \ldots, S_{T}\right\}$ is a given set of integers and $D \in \mathbb{N}$. $\mathcal{I}_{\text {SSP }}$ is a YES-instance for the subsetsum problem if there exists a subset $\mathcal{S}^{\prime}$ of $\mathcal{S}$ such that the elements of $\mathcal{S}^{\prime}$ sum to $D$. Observe that we may assume without loss of generality that $\max _{t}\left(S_{t}\right) \leq \min _{t}\left(X_{t}^{\max }\right)$ since we can scale down $D$ and all elements of $\mathcal{S}$ by a positive constant without changing the problem structure and hardness. Based on $\mathcal{I}_{\text {SSP }}$, we construct an instance $\mathcal{I}_{(P)}$ of $(P)$ with $\vec{X}^{\text {max }}$ as maximum charging powers as follows. We set $C=D$ and for $t \in \mathcal{T}$, we choose $X_{t}^{\min }=S_{t}$ and $p_{t}=-\frac{1}{2} S_{t}$. Note that $\mathcal{I}_{(P)}$ is a valid instance of $(P)$ since for all $t \in \mathcal{T}$ we have $X_{t}^{\min }=S_{t} \leq \max _{t}\left(S_{t}\right) \leq \min _{t}\left(X_{t}^{\max }\right) \leq X_{t}^{\max }$.
We now show that $\mathcal{I}_{\text {SSP }}$ is a YES-instance for the subset-sum problem if and only if $\mathcal{I}_{(P)}$ has an optimal objective value of $\sum_{t=1}^{T} \frac{1}{4} S_{t}^{2}$. First, if $\mathcal{I}_{\mathrm{SSP}}$ is a YES-instance, then the solution

$$
\left(x_{t}^{\prime}, y_{t}^{\prime}\right)= \begin{cases}\left(S_{t}, 1\right) & \text { if } S_{t} \in \mathcal{S}^{\prime} \\ (0,0) & \text { otherwise }\end{cases}
$$

is feasible for $\mathcal{I}_{(P)}$. Since for all $t \in \mathcal{T}$ it holds that $f_{t}(0)=\left(-\frac{1}{2} S_{t}\right)^{2}=\left(\frac{1}{2} S_{t}\right)^{2}=f_{t}\left(S_{t}\right)$, the objective value of this solution is $\sum_{t=1}^{T} \frac{1}{4} S_{t}^{2}$. Note that this is a lower bound on the objective value of $\mathcal{I}_{(P)}$ since for all $t \in \mathcal{T}$ with $X_{t}^{\text {min }}<$ $x_{t} \leq X^{\max }$ it holds that $f_{t}^{\prime}\left(x_{t}\right)=2\left(-\frac{1}{2} S_{t}+x_{t}\right)>S_{t}>0$ and thus $f_{t}\left(x_{t}\right)>f\left(X_{t}^{\min }\right)=\frac{1}{4} S_{t}^{2}$. Therefore, $\left(\vec{x}^{\prime}, \vec{y}^{\prime}\right)$ is also optimal for $\mathcal{I}_{(P)}$. On the other hand, if $\mathcal{I}_{(P)}$ has an optimal solution $\left(\vec{x}^{*}, \vec{y}^{*}\right)$ with objective value $\sum_{t=1}^{T} \frac{1}{4} S_{t}^{2}$, then we must have that $x_{t}^{*}=0$ (when $y_{t}^{*}=0$ ) or $x_{t}^{*}=X_{t}^{\text {min }}=S_{t}$ (when $\left.y_{t}^{*}=1\right)$ for all $t \in \mathcal{T}$. Let $\mathcal{S}^{\prime \prime}:=\left\{X_{t}^{\min } \mid y_{t}^{*}=1\right\}$ and note that $\mathcal{S}^{\prime \prime} \subseteq \mathcal{S}$ by construction of $\mathcal{I}_{(P)}$. Since $\left(\vec{x}^{*}, \vec{y}^{*}\right)$ is feasible for $\mathcal{I}_{(P)}$, it follows that $\sum_{x \in \mathcal{S}^{\prime \prime}} x=\sum_{i=1}^{T} x_{t}^{*}=C=D$. Thus, the sum of all elements in $\mathcal{S}^{\prime \prime}$ is equal to $D$ and $\mathcal{I}_{\text {SSP }}$ is a YES-instance of the subset-sum problem. Since the reduction of $\mathcal{I}_{\text {SSP }}$ to $\mathcal{I}_{(P)}$ can be done in polynomial time, we may conclude that $(P)$ is NP-hard.

The proof of Lemma 1 suggests that the difficulty of Problem $(P)$ might be due to the fact that the lower bounds $X_{t}^{\text {min }}$ are not the same. Therefore, in the remainder of this paper, we consider only problems $(P)$ where all lower bounds are equal, i.e., we replace Constraint (1) by $X^{\min } y_{t} \leq x_{t} \leq X_{t}^{\max } y_{t}$ for all $t \in \mathcal{T}$ and some $X^{\text {min }} \in \mathbb{R}_{+}$.

One question that remains is whether this new version of Problem $(P)$ is still NP-hard. To this end, we introduce two subclasses $\mathcal{P}_{1}$ and $\mathcal{P}_{2}$ of problem instances of $(P)$. Each of these classes respectively consists of all instances for which:

Class 1. $X_{i}^{\max } \leq X_{j}^{\max }$ implies $p_{i}>p_{j}$ (and thus $i<j$ ).

Class 2. $X_{t}^{\max } \geq 2 X^{\min }$ for all $t \in \mathcal{T}$.

In the remainder of this paper, we only consider instances of Problem $(P)$ that belong to the subclasses $\mathcal{P}_{1}$ or $\mathcal{P}_{2}$. In Section III, we derive an $O(T \log (T))$ time algorithm for $(P)$ restricted to these instances. We come back to the necessity of this for our solution approaches in Section III.

\section{ANALYSIS AND INITIAL SOLUTION APPROACH}

In this section, we analyze the structure of Problem $(P)$ and its optimal solutions. Based on this, we derive a first solution approach for $(P)$ that runs in $O\left(T^{2}\right)$ time. In Section IV, we apply the analysis in the current section to derive an $O(T \log (T))$ time algorithm.

The main result of this section is that there exists an optimal solution to Problem $(P)$ wherein the first intervals are exactly those wherein no charging or charging at $X^{\mathrm{min}}$ is done:

Lemma 2. There exists an optimal solution $\vec{x}$ to Problem $(P)$ and integers $K, J \in\{0, \ldots, T\}$ with $K \leq J$ such that

- $x_{t}=0$ for $t \leq K$,

- $x_{t}=X^{\min }$ for $K+1 \leq t \leq J$,

- $X^{\min }<x_{t} \leq X_{t}^{\max }$ for $J+1 \leq t \leq T$. 
Proof: Suppose that there exists no optimal solution that has the structure described in the statement of this lemma and let $\vec{x}^{*}$ be an optimal solution to Problem $(P)$. We prove the lemma by contradiction by showing that $\vec{x}^{*}$ cannot be optimal. For this, we first define $\vec{x}^{\prime}(i, j, \epsilon)$ to be the solution obtained from $\vec{x}^{*}$ by moving a load of $\epsilon>0$ from interval $i$ to interval $j$. Moreover, for any feasible solution $\vec{x}$, let $V(\vec{x})$ be the objective value of $(P)$ for $\vec{x}$. If $\vec{x}^{\prime}(i, j, \epsilon)$ is feasible, then the difference $V\left(\vec{x}^{*}\right)-V\left(\vec{x}^{\prime}(i, j, \epsilon)\right)$ is equal to

$$
\begin{aligned}
& \left(p_{i}+x_{i}^{*}\right)^{2}+\left(p_{j}+x_{j}^{*}\right)^{2}-\left(p_{i}+x_{i}^{*}-\epsilon\right)^{2}-\left(p_{j}+x_{j}^{*}+\epsilon\right)^{2} \\
& =2 \epsilon\left(p_{i}+x_{i}^{*}-p_{j}-x_{j}^{*}-\epsilon\right) .
\end{aligned}
$$

We distinguish between two cases. First, suppose that the problem instance is in $\mathcal{P}_{1}$. Then there exist time intervals $s<t$ such that $x_{s}^{*}>x_{t}^{*}$. Note that $\vec{x}^{\prime}\left(s, t, x_{s}^{*}-x_{t}^{*}\right)$, i.e., the solution obtained from $\vec{x}^{*}$ by interchanging $x_{s}^{*}$ and $x_{t}^{*}$, is feasible. It follows from (2) that

$$
V\left(\vec{x}^{*}\right)-V\left(\vec{x}^{\prime}\left(s, t, x_{s}^{*}-x_{t}^{*}\right)\right)=2\left(x_{s}^{*}-x_{t}^{*}\right)\left(p_{s}-p_{t}\right)>0
$$

since $s<t$ implies $p_{s}>p_{t}$ by the ordering of the intervals and $x_{s}^{*}>x_{t}^{*}$. It follows that $V\left(\vec{x}^{\prime}\left(s, t, x_{s}^{*}-x_{t}^{*}\right)\right)<V\left(\vec{x}^{*}\right)$ and thus that $\vec{x}^{*}$ cannot be optimal.

Now suppose that the problem instance is in $\mathcal{P}_{2}$. First, suppose that there exist time intervals $s<t$ such that $X^{\min } \leq x_{s}^{*} \leq X_{s}^{\max }$ and $x_{t}^{*}=0$. If $x_{s}^{*} \leq X_{t}^{\max }$, then $\vec{x}^{\prime}\left(s, t, x_{s}^{*}-x_{t}^{*}\right)$ is feasible and we can use the argument used above to conclude that $\vec{x}^{*}$ cannot be optimal. If $x_{s}^{*}>X_{t}^{\max }$, we have $x_{s}^{*}>2 X^{\text {min }}$ since the instance is in $\mathcal{P}_{2}$. Then $\vec{x}^{\prime}\left(s, t, X^{\mathrm{min}}\right)$, i.e., the solution obtained from $\vec{x}^{*}$ by moving a load of $X^{\mathrm{min}}$ from $s$ to $t$, is feasible and (2) implies that

$V\left(\vec{x}^{*}\right)-V\left(\vec{x}^{\prime}\left(s, t, X^{\min }\right)\right)=2 X^{\min }\left(p_{s}+x_{s}^{*}-p_{t}-X^{\min }\right)>0$ since $x_{s}^{*} \geq X^{\mathrm{min}}$ and $p_{s}>p_{t}$. It follows that $\vec{x}^{*}$ is not optimal.

Second, suppose that there exists intervals $s<t$ such that $X^{\min }<x_{s}^{*} \leq X_{s}^{\max }$ and $x_{t}^{*}=X^{\mathrm{min}}$. Choose $\epsilon^{\prime}>0$ such that $\epsilon^{\prime}<x_{s}^{*}-X^{\min }$. Then $\vec{x}^{\prime}\left(s, t, \epsilon^{\prime}\right)$ is feasible and (2) implies that $V\left(\vec{x}^{*}\right)-V\left(\vec{x}^{\prime}\left(s, t, \epsilon^{\prime}\right)\right)>0$. Thus, $\vec{x}^{*}$ is not optimal.

Let $\left(P^{K}\right)$ the problem of solving Problem $(P)$ where the value $K$ from Lemma 1 is given, i.e., solving Problem $(P)$ with the additional constraint $\sum_{i=1}^{T} y_{t}=K$. Lemma 1 implies that we can find an optimal solution to $\left(P^{K}\right)$, assuming that $\left(P^{K}\right)$ is feasible, by setting $y_{t}^{*}=0$ and $x_{t}^{*}=0$ for $t \leq K$, setting $y_{t}^{*}=1$ for $t>K$, and solving the remaining problem:

$$
\begin{aligned}
\left(P^{K}\right): \min _{x_{K+1}, \ldots, x_{T}} & \sum_{t=K+1}^{T} f_{t}\left(x_{t}\right)=\sum_{t=K+1}^{T}\left(p_{t}+x_{t}\right)^{2} \\
\text { s.t. } & \sum_{t=K+1}^{T} x_{t}=C, \\
& X^{\min } \leq x_{t} \leq X_{t}^{\max }, \quad t=K+1, \ldots, T .
\end{aligned}
$$

Problem $\left(P^{K}\right)$ is a standard quadratic resource allocation problem that can be solved efficiently [13]. In particular, it can be solved in $O(T-K)$ time (see, e.g., the algorithms in [14]). Thus, we can solve Problem $(P)$ in $O\left(T^{2}\right)$ time by solving $\left(P^{K}\right)$ for $0 \leq K \leq T-1$ and selecting the solution with the lowest objective value. However, we can improve this time complexity by solving each subproblem $\left(P^{K}\right)$ using the solution of the previous subproblem $\left(P^{K-1}\right)$ as input. In Section IV, we propose an $O(T \log (T))$ time algorithm that solves Problem $(P)$ in this way.

\section{An $O(T \log (T))$ ALgORITHM FOR $(P)$}

In this section, we first analyze the properties of the optimal solution to $\left(P^{K}\right)$ in Section IV-A. In particular, we show that this optimal solution can be characterized by two values. In Section IV-B, we show how we can use these values to solve $\left(P^{K+1}\right)$ using the optimal solution to $\left(P^{K}\right)$ and in Section IV-C we present an $O(T \log (T))$ time algorithm to solve Problem $(P)$.

\section{A. Characterization of optimal solutions to $\left(P^{K}\right)$}

Let $\left(\left(\vec{x}^{K}\right)^{*},\left(\vec{y}^{K}\right)^{*}\right)$ denote the optimal solution to $\left(P^{K}\right)$. We first characterize the optimal activation decision $\left(\vec{y}^{K}\right)^{*}$. Lemma 1 implies that $\left(y_{t}^{K}\right)^{*}=0$ for $t \leq K$ and $\left(y_{t}^{K}\right)^{*}=1$ for $t>K$. Note that this implies that $\left(y_{t}^{K}\right)^{*}=0$ if $p_{t} \geq$ $p_{K}$ and $\left(y_{t}^{K}\right)^{*}=1$ if $p_{t}<p_{K}$. We can thus translate the characterization of $\left(\vec{y}^{K}\right)^{*}$ by $K$ into a characterization by $\vec{p}$. More precisely, there exists a value $Z_{K}^{\text {act }}$, which we call the activiation level, such that

$$
\left(y_{t}^{K}\right)^{*}= \begin{cases}1 & \text { if } p_{t}+X^{\mathrm{min}} \leq Z_{K}^{\text {act }} \\ 0 & \text { otherwise }\end{cases}
$$

The interpretation of $Z_{K}^{\text {act }}$ and (3) is that we only charge in a given interval if the resulting total load in that interval does not exceed $Z_{K}^{\text {act }}$. Since the minimum allowed charging rate is $X^{\mathrm{min}}$, Rule (3) exactly represents this policy. It follows that defining $Z_{K}^{\text {act }}$ as

$$
Z_{K}^{\text {act }}:=p_{K+1}+X^{\mathrm{min}}
$$

ensures that $\left(y_{t}^{K}\right)^{*}=0$ if and only if $t \leq K$.

We now characterize $\left(\vec{x}^{K}\right)^{*}$. Note that Rule (3) already characterizes $\left(x_{t}^{K}\right)^{*}$ for $t \leq K$ since in that case $\left(x_{t}^{K}\right)^{*}=0$. It is well-known that the optimal solution to $\left(P^{K}\right)$ can be characterized by a single value $Z_{K}^{\text {fill }}$, known as the fill-level (see, e.g., [13], [15], [16]), as follows:

$$
\left(x_{t}^{K}\right)^{*}= \begin{cases}X^{\min } & \text { if } p_{t}+X^{\min } \geq Z_{K}^{\text {fill }}, \\ Z_{K}^{\text {fill }}-p_{t} & \text { if } p_{t}+X^{\min }<Z_{K}^{\text {fill }}<p_{t}+X_{t}^{\max }, \\ X_{t}^{\max } & \text { if } p_{t}+X_{t}^{\max } \leq Z_{K}^{\text {fill }} .\end{cases}
$$

The interpretation of $Z_{K}^{\text {fill }}$ and Rule (5) is that, ideally, the base load $p_{t}$ is filled up with EV charging to $Z_{K}^{\text {fill }}$. If the combined base load and charging profile already exceeds $Z_{K}^{\text {fill }}$ when charging at the smallest allowed rate $X^{\mathrm{min}}$, we charge at this minimum rate. Similarly, if the combined profile does not exceed $Z_{K}^{\text {fill }}$ even when charging is done at the maximum rate $X_{t}^{\max }$, we charge at this maximum rate.

We define the lower and upper critical values of an interval $t$ as $\lambda_{t}:=p_{t}+X^{\mathrm{min}}$ and $\mu_{t}:=p_{t}+X_{t}^{\max }$ respectively. Given $Z_{K}^{\text {act }}$ and $Z_{K}^{\text {fill }}$, one can now compute the optimal charging schedule $\left(\vec{x}^{K}\right)^{*}$ directly by combining (3) and (5) as follows:

$$
\left(x_{t}^{K}\right)^{*}=u\left(Z_{K}^{\text {act }}-\lambda_{t}\right) \cdot \max \left(X^{\min }, \min \left(Z_{K}^{\text {fill }}+p_{t}, X_{t}^{\max }\right)\right),
$$




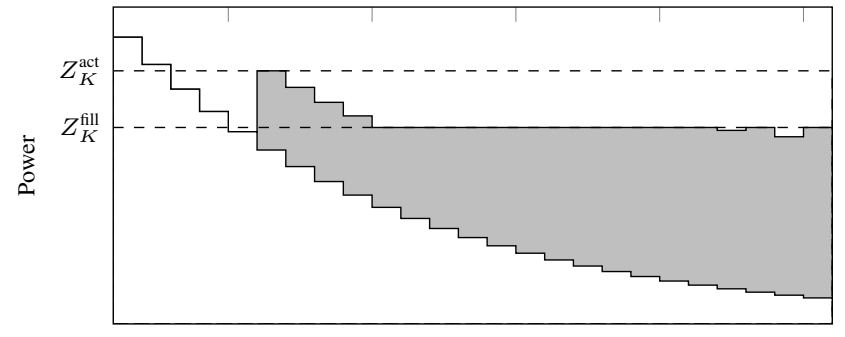

Time interval

Base load $\square$ EV charging

Fig. 1. Illustrative example of the characterization by $Z^{\text {act }}$ and $Z^{\text {fill }}$ in (6).

where $u(\cdot)$ is the unit step function that is 1 if its argument is nonnegative and 0 otherwise. Fig. 1 visualizes the characterization of $\vec{x}^{*}$ by $Z_{K}^{\text {act }}$ and $Z_{K}^{\text {fill }}$ for $K=5$. One important consequence of the characterization by (6) is that in order to solve $\left(P^{K}\right)$, we only need to compute $Z_{K}^{\text {act }}$ and $Z_{K}^{\text {fill }}$ and use (6) to derive from these values the optimal solution. Note that this implies that we can characterize also the optimal solution to Problem $(P)$ by two levels $Z^{\text {act }}$ and $Z^{\text {fill }}$.

Given $K$, we can compute $Z_{K}^{\text {act }}$ directly from the definition in (4). Thus, the remaining question is how to compute $Z_{K}^{\text {fill }}$. In Section IV-B, we show how $Z_{K+1}^{\text {fill }}$ can be computed from $Z_{K}^{\text {fill }}$.

B. Computing $Z_{K+1}^{\text {fill }}$ from $Z_{K}^{\text {fill }}$

Fig. 2 shows an example instance of Problem $(P)$. More precisely, Fig. $2 \mathrm{a}$ and $2 \mathrm{~b}$ show the optimal solution and characterization of $\left(P^{K}\right)$ and $\left(P^{K+1}\right)$ respectively. The figures imply the following approach to compute $Z_{K+1}^{\text {fill }}$ and solve $\left(P^{K+1}\right)$ using $Z_{K}^{\text {fill }}$. We can construct an optimal solution to $\left(P^{K+1}\right)$ by redistributing the load of interval $K$ over the intervals $K+1, \ldots, T$, on top of the optimal solution to $\left(P^{K}\right)$. We do this by setting $Z^{\text {fill }}:=Z_{K}^{\text {fill }}$ and raising $Z^{\text {fill }}$ until it is high enough to accommodate the extra load (see Fig. 2b). In other words, we find a new, higher level $Z_{K+1}^{\text {fill }}$ such that the corresponding solution $\left(\vec{x}^{K+1}\right)^{*}$ as computed by (5) is feasible, i.e., sums to $C$. We can do this using a standard valley-filling approach, starting from the fill-level $Z^{\text {fill }}=Z_{K}^{\text {fill }}$. Note that we only have to do this if $K \neq J$, i.e., when the load of interval $K$ is $X^{\text {min }}$, since otherwise the optimal objective value of the subsequent subproblems can only increase (see also [13]).

We now briefly describe the valley-filling approach. Note that (5) implies that when we increase $Z^{\text {fill }}$ by a small amount, only the load of those intervals $t$ with $\lambda_{t}<Z^{\text {fill }}<\mu_{t}$ increases. We call these intervals free and define the set of free intervals as $\mathcal{F}:=\left\{t \in \mathcal{T} \mid \lambda_{t}<Z^{\text {fill }}<\mu_{t}\right\}$ and let $F:=|\mathcal{F}|$.

Given the current fill-level $Z^{\text {fill }}$, we first check if we can equally distribute the remaining to-be-redistributed amount $\Delta$ of interval $K$ over the intervals in $\mathcal{F}$ such that the resulting fill-level does not exceed any of the critical values. That is, given that $\nu$ is the smallest critical value that is larger than $Z^{\text {fill }}$, we check if $Z^{\text {fill }}+\Delta / F \leq \nu$. If this is the case, we set $Z_{K+1}^{\text {fill }}=Z^{\text {fill }}+\Delta / F$ and we are done. On the other hand, if $\nu$ is exceeded, we distribute an amount of $F\left(\nu-Z^{\text {fill }}\right)$ over the

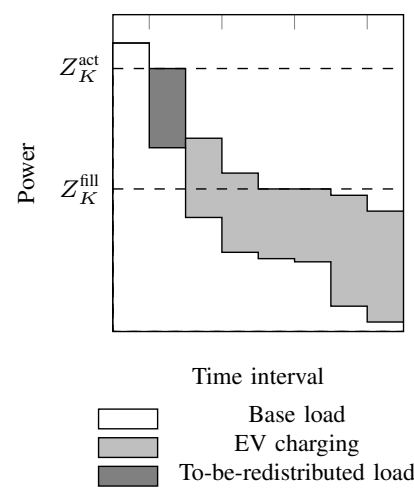

(a) Optimal solution to $\left(P^{K}\right)$.

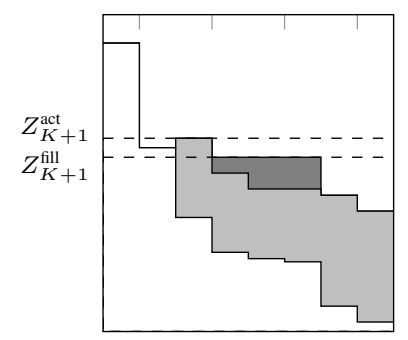

Time interval

Base load EV charging Redistributed load

(b) Optimal solution to $\left(P^{K+1}\right)$.
Fig. 2. Relation between $Z_{K}^{\text {fill }}$ and $Z_{K+1}^{\text {fill }}$.

free intervals and set $Z^{\text {fill }}$ to $\nu$. If $\nu$ is a lower critical value, the corresponding time interval becomes free when $Z^{\text {fill }} \geq \nu$ and thus we add it to $\mathcal{F}$. If $\nu$ is an upper critical value, the load on the corresponding interval reaches its upper bound and we remove it from $\mathcal{F}$. We have now obtained a similar problem, namely to redistribute an amount of $\Delta-F\left(\nu-Z^{\text {fill }}\right)$ over the intervals $K+1, \ldots, T$. Thus, we can update $\Delta$ and repeat the above procedure until all the to-be-redistributed load can be equally distributed over the free intervals.

If we store $\mathcal{F}$ as a vector sorted according to ascending values of $\mu_{t}$ and preserve this property when inserting and removing time intervals in and from $\mathcal{F}$, we can compute $\nu$ as follows, given $K, J$ and $\mathcal{F}$ :

$$
\nu:=\min \left(\min _{K+1 \leq t \leq J} \lambda_{t}, \min _{t \in \mathcal{F}} \mu_{t}\right)=\min \left(\lambda_{J}, \mu_{\mathcal{F}_{1}}\right),
$$

where $\mathcal{F}_{1}$ is the first element of the sorted vector $\mathcal{F}$. Note that this can be done in $O(1)$ time. Also, note that when an interval is added to $\mathcal{F}$, we must reduce $J$ by 1 . That is, the number of time intervals whose load is $X^{\text {min }}$ under the new fill-level reduces by 1 .

\section{An $O(T \log (T))$ time algorithm}

Algorithm 1 summarizes the approach presented in this section. To improve its efficiency, we compute the objective value of each subproblem $\left(P^{K}\right)$ by storing the objective value generated by all intervals that are not in $\mathcal{F}$ in the scalar $N$. As a consequence, we can efficiently compute the objective value $V^{K}$ of each subproblem $\left(P^{K}\right)$ after computing $Z_{K}^{\text {fill }}$.

We conclude this section by establishing the time complexity of Algorithm 1. Note that the inner while-loop (Lines 8-16) is executed at most $2 T$ times during the entire course of the algorithm since the number of critical values is $2 T$. Since the insertion of $J$ in the sorted vector $\mathcal{F}$ in Line 11 can be done in $O(\log (F))$ time using binary search, each iteration of this while-loop takes $O(\log (T))$ time. As a consequence, the outer while-loop (Lines 7-20) takes $O(T \log (T))$ time. The remaining part of the algorithm also takes $O(T \log (T))$ time due to the sorting in Lines 1 and 23. It follows that the time complexity of Algorithm 1 is $O(T \log (T))$. 


\section{AN ONLINE ALGORITHM}

In this section, we use the analysis of Section IV-A to derive an online algorithm for Problem $(P)$ when the base load profile $\vec{p}$ is unknown beforehand. This algorithm is similar to existing online algorithms for other types of EV charging problems (see, e.g., [15]-[18]).

The characterization of optimal solutions by $Z^{\text {act }}$ and $Z^{\text {fill }}$ and the rule in (6) gives rise to the following online algorithm. Suppose that we have predictions $\hat{Z}^{\text {act }}$ and $\hat{Z}^{\text {fill }}$ of $Z^{\text {act }}$ and $Z^{\text {fill }}$ respectively. We can compute an approximate solution $\overrightarrow{\hat{x}}$ by adapting the decision rule in (6):

$$
\hat{x}_{t}=u\left(\hat{Z}^{\text {act }}-\lambda_{t}\right) \cdot \max \left(X^{\min }, \min \left(\hat{Z}^{\text {fill }}-p_{t}, X_{t}^{\max }\right)\right) .
$$

Note that the computation of $\hat{x}_{t}$ can be postponed until the
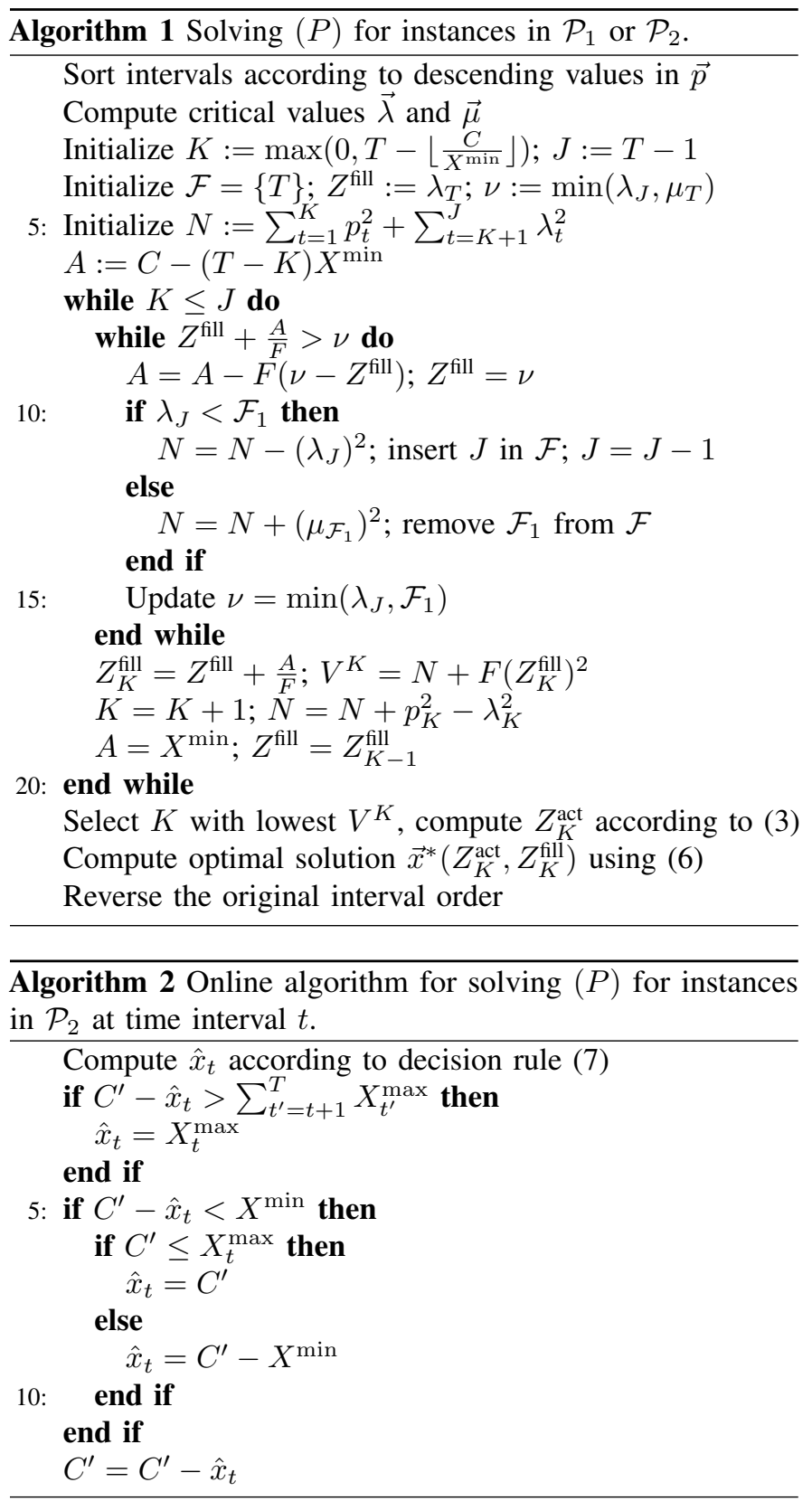

start of interval $t$, i.e., until $p_{t}$ becomes available or can be predicted more accurately.

After determining the online decision $\hat{x}_{t}$, we must check whether there exists a feasible schedule for the future intervals such that the charging requirement $C$ is met. For this, let $C^{\prime}$ be the amount that still must be charged from interval $t$ on. If the problem instance is in $\mathcal{P}_{2}$, it follows that a feasible future schedule exists if $X^{\mathrm{min}} \leq C^{\prime}-\hat{x}_{t} \leq \sum_{t^{\prime}=t+1}^{T} X_{t^{\prime}}^{\max }$ (see also [19]). If $C^{\prime}-\hat{x}_{t}>\sum_{t^{\prime}=t+1}^{T} X_{t^{\prime}}^{\max }$, we set $\hat{x}_{t}$ to $X_{t}^{\max }$. Subsequently, if $C^{\prime}-\hat{x}<X^{\text {min }}$, then we either charge all remaining load $C^{\prime}$ in the current interval if this is feasible, i.e., if $C^{\prime} \leq X_{t}^{\max }$, and otherwise we charge $C^{\prime}-X^{\mathrm{min}}$ and leave an amount of $X^{\mathrm{min}}$ to be charged during one of the future intervals. Since for instances in $\mathcal{P}_{1}$ we cannot derive a feasibility condition in the form of an allowed interval like we did for instances in $\mathcal{P}_{2}$ [19], we only consider instances in $\mathcal{P}_{2}$.

Algorithm 2 captures the approach presented in this section. The main advantage of this method is that no prediction of $\vec{p}$ is required beforehand. Moreover, as we show in Section VI, errors in the prediction of $Z^{\text {act }}$ and $Z^{\text {fill }}$ do not lead to large deviations in the objective value of the online solution $\overrightarrow{\hat{x}}$.

\section{Evaluation}

This section evaluates the performance of the offline and online algorithm derived in the previous sections. For the offline algorithm, we assess its efficiency and for the online algorithm, we validate its accuracy and the predictability of the two characterizing levels. All simulations and computations in this section are executed on a Dell Inspiron 15 with an Intel Core i7-6700HQ CPU at $2.60 \mathrm{GHz}$ and $16 \mathrm{~GB}$ of RAM.

We apply the offline and online approaches to schedule an EV belonging to a household in southern Germany. We assume that the EV is charged between 19:00 PM and 7:00 AM and that this charging window is divided into 15-minute time intervals. We assume that the base load profile $\vec{p}$ is not known on forehand. We choose the Nissan Leaf as reference EV [20], meaning that we set $C=40 \mathrm{kWh}$. Furthermore, we assume that a home charger with a maximum charging rate of $X_{t}^{\max }=6.6 \mathrm{~kW}$ for all $t \in \mathcal{T}$ is used and we set $X^{\min }=1.1 \mathrm{~kW}$, which is in line with the recommendations in [7].

We generate predictions of $Z^{\text {act }}$ and $Z^{\text {fill }}$ by computing the optimal EV schedule for 70 previous days under the same circumstances as for the current charging session, i.e., the same values of $C, X^{\text {min }}$ and $\vec{X}^{\text {max }}$, using the house power profile of each of the days respectively. In line with the findings in [21], we expect that the resulting predictions properly represent the behavior of $Z^{\text {act }}$ and $Z^{\text {fill }}$. For each previous day, we compute an online solution using Algorithm 2 and the predictions corresponding to that day as input. We compare each resulting solution to the optimal solution of the current charging session by computing its relative objective value as the ratio between the objective value of the solution and the optimal objective value. Note that the smallest possible relative objective value is 1 , which occurs when the online solution is optimal.

Fig. 3 shows the relative objective value of the online solution for different choices of predictions $\hat{Z}^{\text {act }}$ and $\hat{Z}^{\text {fill }}$. Here, the 


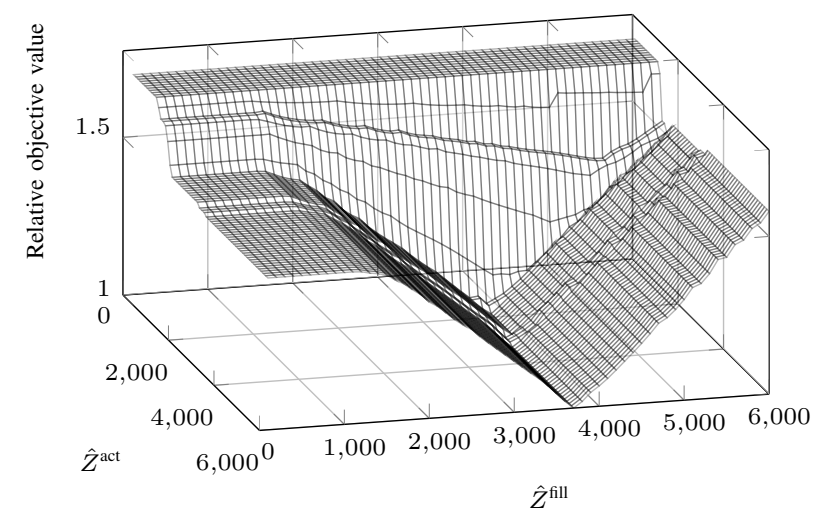

Fig. 3. Relative objective value for different combinations of $Z^{\text {act }}$ and $Z^{\text {fill }}$ for one day. The minimum of 1 occurs at $\left(\hat{Z}^{\text {act }}, \hat{Z}^{\text {fill }}\right)=(3206,3705)$.

TABLE I

Simulation RESUlts. A RELATIVE OBJECTIVE VALUE OF 1 IMPLIES THAT THE ONLINE SOLUTION IS OPTIMAL.

\begin{tabular}{l|rrrr}
\hline & Minimum & Maximum & Mean & Median \\
\hline$Z^{\text {act }}$ & 1595 & 3947 & 2707 & 2954 \\
$Z^{\text {fill }}$ & 3474 & 3761 & 3611 & 3615 \\
Relative objective value & 1.0001 & 1.3770 & 1.0679 & 1.0339 \\
Running time $\left(10^{-4} \mathrm{~s}\right)$ & 1.63 & 3.26 & 2.01 & 1.80 \\
\hline
\end{tabular}

minimum is attained at $\left(Z^{\text {act }}\right)^{*}=3206$ and $\left(Z^{\text {fill }}\right)^{*}=3705$. The figure implies that slight deviations of $\hat{Z}^{\text {act }}$ and $\hat{Z}^{\text {fill }}$ from $\left(Z^{\text {act }}\right)^{*}$ and $\left(Z^{\text {fill }}\right)^{*}$ respectively hardly influence the relative objective value. This implies that the online approach is robust against prediction errors in these two levels.

Table I provides the results of the simulation study. Since $Z^{\text {act }}$ varies significantly over the course of consecutive days and $Z^{\text {act }}$ remains within a small interval around $\left(Z^{\text {fill }}\right)^{*}$, these results imply that $Z^{\text {fill }}$ is easier to predict than $Z^{\text {act }}$. The objective value of the online solution is at most $3.39 \%$ higher than the optimal objective value for $50 \%$ of the days, which implies that the online algorithm often computes online solutions that are near-optimal. Finally, the last row of Table I implies that our offline algorithm is very fast.

\section{CONCLUSIONS}

In this paper, we presented an efficient algorithm for scheduling of EV charging in DEM that takes into account a minimum charging threshold and flattens the combined base load and EV profile. Moreover, we derived an online algorithm that does not require a prediction of the base load profile but only the prediction of two values. This algorithm is robust against prediction errors in these values and is able to produce near-optimal solutions.

One direction for future research is to integrate our algorithms into an existing DEM system and evaluate their performance. Another interesting direction is to extend the current work to develop a scheduling algorithm for home batteries and EVs with vehicle-to-grid options that takes into account the minimum-threshold constraint. Finally, the online approach can be improved by further analysis on its robustness and by developing sophisticated prediction approaches for the two characterizing values.

\section{ACKNOWLEDGMENT}

This research is conducted within the SIMPS project (647.002.003) supported by NWO and Eneco, and the EASI project (12700) supported by STW and Alliander.

\section{REFERENCES}

[1] International Energy Agency, "Global EV Outlook 2018: Towards crossmodal electrification." [Online]. Available: https://webstore.iea.org/globalev-outlook-2018. Retrieved on September 5, 2018.

[2] P. Siano, "Demand response and smart grids - a survey," Renewable and Sustainable Energy Reviews, vol. 30, pp. 461-478, 2014.

[3] M. E. T. Gerards, H. A. Toersche, G. Hoogsteen, T. van der Klauw, J. L. Hurink, and G. J. M. Smit, "Demand side management using profile steering," in 2015 IEEE Eindhoven PowerTech, 2015.

[4] J. C. Mukherjee and A. Gupta, "A review of charge scheduling of electric vehicles in smart grid," IEEE Systems Journal, vol. 9, no. 4, pp. 1541-1553, 2015.

[5] K. Young, C. Wang, L. Y. Wang, and K. Strunz, "Electric vehicle battery technologies," in Electric vehicle integration into modern power networks, R. Garcia-Valle and J. A. Peças Lopes, Eds. New York: Springer, 2013, ch. 2, pp. 15-56.

[6] IEC-61851-1, "Electric vehicle conductive charging system - Part 1: General requirements," 2017.

17] E. Apostolaki-Iosifidou, P. Codani, and W. Kempton, "Measurement of power loss during electric vehicle charging and discharging," Energy, vol. 127, pp. 730-742, 2017.

[8] A. Di Giorgio, F. Liberati, and S. Canale, "Electric vehicles charging control in a smart grid: A model predictive control approach," Control Engineering Practice, vol. 22, pp. 147-162, 2014.

[9] F. Javed, N. Arshad, F. Wallin, I. Vassileva, and E. Dahlquist, "Forecasting for demand response in smart grids: an analysis on use of anthropologic and structural data and short term multiple loads forecasting," Applied Energy, vol. 96, pp. 150-160, 2012.

[10] S. Michel, N. Perrot, and F. Vanderbeck, "Knapsack problems with setups," European Journal of Operational Research, vol. 196, no. 3, pp. 909-918, 2009.

[11] X. Sun, X. Zheng, and D. Li, "Recent advances in mathematical programming with semi-continuous variables and cardinality constraint," Journal of the Operations Research Society of China, vol. 1, no. 1, pp. 55-77, 2013.

[12] L. Meussen, "Electrical vehicle charging under two types of steering signals with a lower bound," M.Sc. thesis, Radboud University Nijmegen, 2015.

[13] M. Patriksson and C. Strömberg, "Algorithms for the continuous nonlinear resource allocation problem - new implementations and numerical studies," European Journal of Operational Research, vol. 243, no. 3, pp. 703-722, 2015.

[14] K. C. Kiwiel, "Breakpoint searching algorithms for the continuous quadratic knapsack problem," Mathematical Programming, vol. 112, no. 2, pp. 473-491, 2008.

[15] N. Chen, C. W. Tan, and T. Q. S. Quek, "Electric vehicle charging in smart grid: optimality and valley-filling algorithms," IEEE Journal of Selected Topics in Signal Processing, vol. 8, no. 6, pp. 1073-1083, 2014.

[16] Y. Mou, H. Xing, Z. Lin, and M. Fu, "Decentralized optimal demand-side management for PHEV charging in a smart grid," IEEE Transactions on Smart Grid, vol. 6, no. 2, pp. 726-736, 2015.

[17] M. E. T. Gerards and J. L. Hurink, "Robust peak-shaving for a neighborhood with electric vehicles," Energies, vol. 9, no. 8(594), 2016

[18] M. H. H. Schoot Uiterkamp, "Robust planning of electric vehicle charging," M.Sc. thesis, University of Twente, 2016.

[19] R. Diao, Y.-F. Liu, and Y.-H. Dai, "A new fully polynomial time approximation scheme for the interval subset sum problem," Journal of Global Optimization, vol. 68, no. 4, pp. 749-775, 2017.

[20] "New Nissan Leaf prices and specifications." [Online]. Available: https://www.nissan.co.uk/vehicles/new-vehicles/leaf/pricesspecifications.html. Retrieved on September 5, 2018.

[21] M. H. H. Schoot Uiterkamp, M. E. T. Gerards, and J. L. Hurink, "Fill-level prediction in online valley-filling algorithms for electric vehicle charging," Accepted for 2018 IEEE PES Innovative Smart Grid Technologies Europe, Sarajevo, 2018. 